\title{
Present Status of the New Multi-Frequency ECRH System for ASDEX Upgrade
}

\author{
Dietmar Wagner, Gerhard Grünwald, Fritz Leuterer, Adriano Manini, Francesco Monaco, Max \\ Münich, Harald Schütz, Jörg Stober, Hartmut Zohm, Thomas Franke, Manfred Thumm, Fellow, IEEE, \\ Roland Heidinger, Gerd Gantenbein, Andreas Meier, Walter Kasparek, Carsten Lechte, Alexander \\ Litvak, Gregory Denisov, Alexei Chirkov, Evgeny Tai, Leonid Popov, Vadim Nichiporenko, Vadim \\ Myasnikov, Elena Solyanova, Sergey Malygin, Fernando Meo, Paul Woskov
}

\begin{abstract}
A new multi-frequency ECRH-System is under construction at the ASDEX Upgrade tokamak experiment. This system employs, for the first time in a fusion device, multifrequency gyrotrons, step-tunable in the range 105-140 GHz. In its final stage the system will consist of 4 gyrotrons with a total power of $4 \mathrm{MW}$ and a pulse length of $10 \mathrm{~s}$. The first two gyrotrons, working at 105 and $140 \mathrm{GHz}$, were installed and tested. Transmission line elements, such as corrugated waveguides, polarizer mirrors and vacuum windows, are designed to cope for this frequency band. The system includes fast steerable launchers at the front end that will allow for localized feedback controlled power deposition in the plasma.
\end{abstract}

Index Terms-Electron Cyclotron Resonance Heating, Steptunable Gyrotron, Fast Steerable Launcher.

\section{INTRODUCTION}

Qingle frequency ECRH systems allow for central power

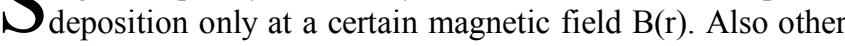
experimental features like the suppression of neoclassical tearing modes (NTM), which requires to drive current on the

Manuscript received August 28, 2007.

D. Wagner, G. Grünwald, F. Leuterer, A. Manini, F. Monaco, M. Münich, J. Stober, H. Zohm and T. Franke are with the Max-Planck-Institut für Plasmaphysik, EURATOM-IPP, Boltzmansstr.2, D-85748 Garching, Germany (phone: 89-3299-2597; fax: 89-3299-1313; e-mail: dietmar.wagner@ipp.mpg.de).

M. Thumm and G. Gantenbein are with the Forschungszentrum Karlsruhe, EURATOM-FZK, Institut für Hochleistungsimpuls- und Mikrowellentechnik, D-76021, Karlsruhe, Germany

R. Heidinger and A. Meier are with the Forschungszentrum Karlsruhe, EURATOM-FZK, Institut für Institut für Materialforschung, D-76021, Karlsruhe, Germany

W. Kasparek and C. Lechte are with the Institut für Plasmaforschung, Universität Stuttgart, D-70569 Stuttgart, Germany

A. Litvak, G.G. Denisov and A.V. Chirkov, are with the Institute of Applied Physics, RAS, 603600 Nizhny Novgorod, Russia

E.M. Tai, L.G. Popov, V.O. Nichiporenko, V.E. Myasnikov, E.A. Solyanova and S.A. Malygin are with GYCOM Ltd. 46 Ulyanov St., Nizhny Novgorod, Russia

F. Meo is with Assocoation EURATOM-Risø National Laboratory, DK4000 Roskilde, Denmark

P. WOSKOV is with the MIT Plasma Science and Fusion Center, Cambridge, MA 02139, U.S.A. high field side, are limited and usually require to adjust the magnetic field accordingly. Those restrictions can be overcome if the gyrotron frequency is variable $[1,2,3]$. The extension of the ECRH operating space in ASDEX Upgrade with respect to the plasma radius (rho p) and the toroidal magnetic field is shown in Figure 1 for the new system with 4 frequencies. In the old $140 \mathrm{GHz}$ ECRH system at ASDEX Upgrade the installed power was only $2 \mathrm{MW}(2 \mathrm{~s})$, of which $1.6 \mathrm{MW}$ was coupled to the plasma. This imposes a limit for current drive, NTM stabilization or generation of internal transport barriers [4]. One of the targets for the new system is therefore an installed power of $4 \mathrm{MW}$ at $140 \mathrm{GHz}$ and a power of around 3 MW at lower frequencies. The pulse length is $10 \mathrm{~s}$ which corresponds to the current diffusion time of several seconds in hot plasmas, like those with an internal transport barrier and $\mathrm{T}_{\mathrm{e}}$ $>10 \mathrm{keV}$. The new system also includes fast movable launcher mirrors. They allow for very localized power deposition such that its center can be feedback controlled, for instance to keep it on a resonant q-surface. In the following, the main components of the new system (gyrotrons, matching optics unit (MOU), transmission line, vacuum windows and launcher) with their peculiarities that are mainly connected to the application of multiple frequencies are described and first experiences are reported.

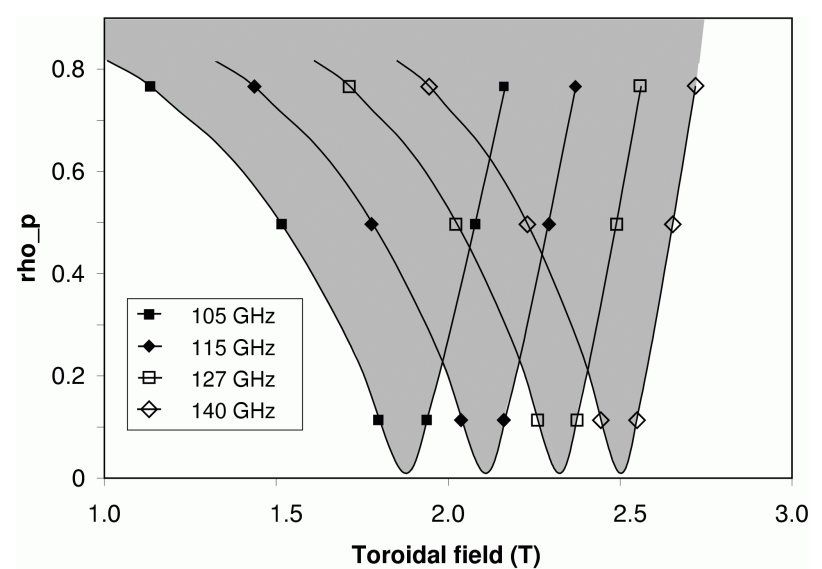

Fig. 1. Extension of the operating space for multi-frequency ECRH at ASDEX Upgrade. 


\section{GYROTRONS}

The two-frequency GYCOM gyrotrons Odissey-1 and Odissey-2 have recently been installed and put into operation. They can work at $105 \mathrm{GHz}$ and at $140 \mathrm{GHz}$. The corresponding operating modes are $\mathrm{TE}_{17,6}$ and $\mathrm{TE}_{22,8}$. Here we make use of the $3 \lambda / 2$ and $4 \lambda / 2$ resonances of the single-disc synthetic diamond vacuum window at these frequencies ( $\lambda$ is the wavelength in diamond). The frequency can be changed by an adjustment of the cryomagnetic field, the gun and collector magnetic fields and operating voltages. The leading time is the variation of the cryomagnetic field and of the beam current adjustment through the filament heating. Both can get near steady state within the 20 minutes time between two ASDEX Upgrade pulses. This was demonstrated up to now with pulses into a load.

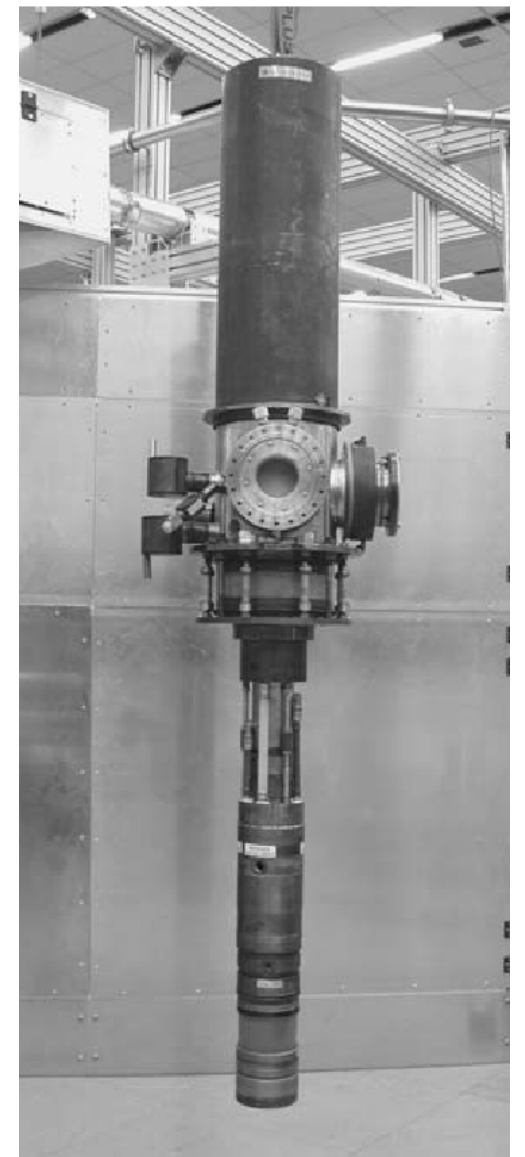

Fig. 2. Two-frequency gyrotron Odissey-1.

The gyrotrons have a single-stage depressed collector. Therefore the cathode voltage can be limited to a maximum value of $60 \mathrm{kV}$. The maximum beam current is $40 \mathrm{~A}$. In this way we can make more efficient use of the existing thyristor controlled high voltage power supplies with capabilities of 70 $\mathrm{kV}$ and $100 \mathrm{~A}$ per unit. This allows us to feed two gyrotrons from one of these units. Nevertheless, there is a separate set of series tetrode and body modulator for each gyrotron, which will allow maximum flexibility for the experimental program (Fig.3). The series modulator is essentially a tetrode (BBC CQK 200-4A) and is used to switch on and off the gyrotron, both for normal operation as well as in an emergency case, for smoothing the ripples of the power supply and for fast modulation of the cathode voltage (up to $25 \mathrm{kHz}$ ). The body modulator is also based on electron tubes (Thales TH5688 and TH5685) and is used for switching on and off the body voltage.

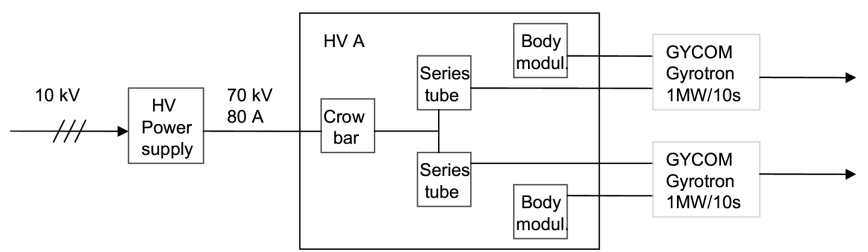

Fig. 3. High voltage power supply scheme.

While the long-pulse testing of the first two-frequency gyrotron Odissey-1 was limited by the available high-power long-pulse load, a new larger GYCOM stainless-steel load allowed for repetitive $10 \mathrm{~s}$ pulses with full power at both frequencies. The load contains no additional absorptive coating. The measured output power of both gyrotrons at $105 \mathrm{GHz}$ and $140 \mathrm{GHz}$ for a pulse length of $10 \mathrm{~s}$ is given in Table 1 . The difference in the output parameters is most likely due to different types of cathodes applied in the gyrotrons.

\begin{tabular}{|l|l|c|c|c|l|}
\hline Frequency & Gyrotron & $\mathrm{U}_{\text {cath }}$ & $\mathrm{U}_{\text {body }}$ & $\mathrm{I}_{\text {beam }}$ & $\begin{array}{l}\text { output } \\
\text { power }\end{array}$ \\
\hline $105 \mathrm{GHz}$ & Odissey-1 & $-44.6 \mathrm{kV}$ & $+24.5 \mathrm{kV}$ & $37.8 \mathrm{~A}$ & $\begin{array}{l}640 \mathrm{~kW} \\
( \pm 10 \%)\end{array}$ \\
\hline $140 \mathrm{GHz}$ & Odissey-2 & $-50.0 \mathrm{kV}$ & $+28.0 \mathrm{kV}$ & $35.0 \mathrm{~A}$ & $\begin{array}{l}638 \mathrm{~kW} \\
( \pm 10 \%)\end{array}$ \\
\hline & Odissey-1 & $-49.0 \mathrm{kV}$ & $+24.0 \mathrm{kV}$ & $40.2 \mathrm{~A}$ & $\begin{array}{l}820 \mathrm{~kW} \\
( \pm 10 \%) \\
890 \mathrm{~kW} \\
( \pm 10 \%)\end{array}$ \\
\hline
\end{tabular}

Table 1 Performance of the two-frequency gyrotrons Odissey-1 and Odissey-2 at ASDEX Upgrade.

For NTM stabilization experiments a fast modulation capability of the gyrotrons is required. This is especially important for future experiments like ITER where the width of the driven EC current will be larger than the marginal island size of the NTM leading to a loss of current drive efficiency in the non-modulated case [5]. Two modulation schemes have been tested with the gyrotrons. A $100 \%$ power modulation up to $0.5 \mathrm{kHz}$ was achieved by switching both, cathode and body voltage on and off. This scheme will be mainly used for heat wave analysis. Higher modulation frequencies up to $25 \mathrm{kHz}$ with modulation depths up to $90 \%$ at $140 \mathrm{GHz}$ were achieved by a reduction of only the cathode voltage to a value where the gyrotron still oscillates while keeping the body voltage constant. If the gyrotron is equipped with a broadband output window it can oscillate at additional frequencies. Figure 4 shows the possible modes and frequencies. The first gyrotron Odissey-1 is currently being equipped with a broadband Brewster CVD-diamond output window and will therefore become step-tunable [6].

The total measured frequency variation during a gyrotron pulse was $140 \mathrm{MHz}$ [7]. Out of this, a drift of $\sim 100 \mathrm{MHz}$ happens in the first $100 \mathrm{~ms}$ of the pulse and repeatedly during on/off 
modulation (Fig.5). This frequency variation contains effects with different time constants. The fastest one is very likely the voltage rise time $(\sim 1 \mathrm{~ms})$ and the slowest one the thermal expansion of the cavity ( $300 \mathrm{~ms})$. Space charge effects and plasma formation in the cavity depend on gas release from the cavity wall and have presumably a time constant in between $[8,9]$. This is different in the case of the modulation only by a reduction of the cathode voltage (Fig.6), where the large frequency drift occurs only at the start of the pulse. During the pulse, only a small frequency pulling (variation of the cathode voltage $U_{\text {cath }}$ ) and a slow frequency drift due to thermal expansion of the cavity wall are observed. Since with this modulation the beam current is not completely switched off, the plasma in the cavity is maintained and a corresponding frequency drift does not occur. This mode of operation allows beam switching via a frequency diplexer (FADIS project [10]) or is also suitable for CTS experiments [7].

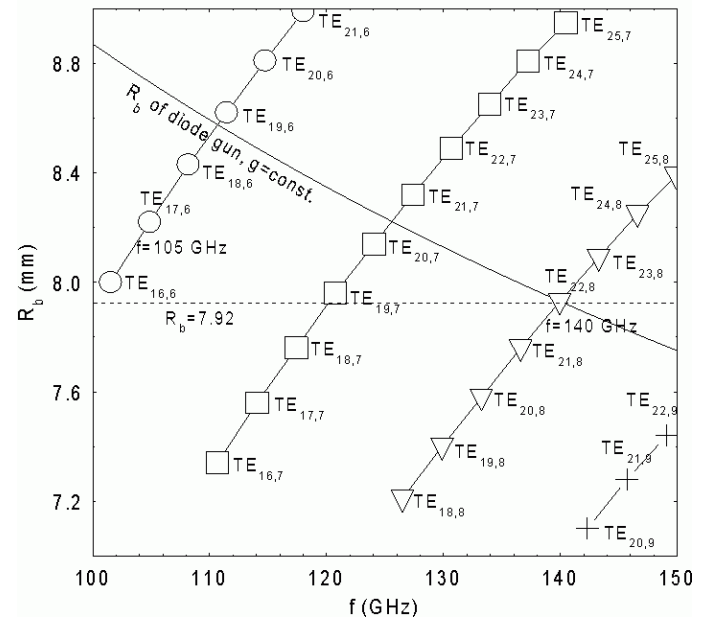

Fig. 4. Electron beam radii and frequencies for operating $\mathrm{TE}_{\mathrm{m}, \mathrm{n}}$ modes of the step-tunable gyrotron. Also shown are curves for constant beam radius $\left(R_{b}=7.92 \mathrm{~mm}\right)$ and $R_{b}$ as a function of frequency for constant pitch factor $(\alpha=$ const.).

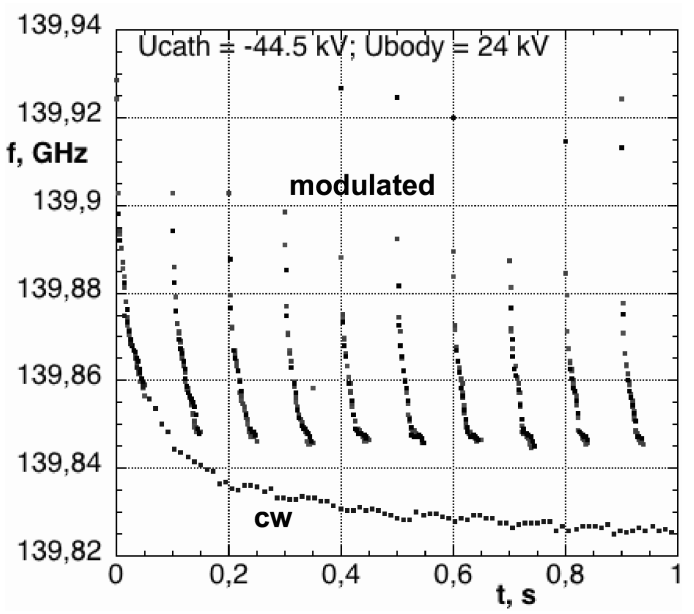

Fig. 5. Measured frequency drift of gyrotron Odissey-1 during a modulated (on/off $50 \mathrm{~ms} / 50 \mathrm{~ms}$ ) and a cw $140 \mathrm{GHz}$ pulse.

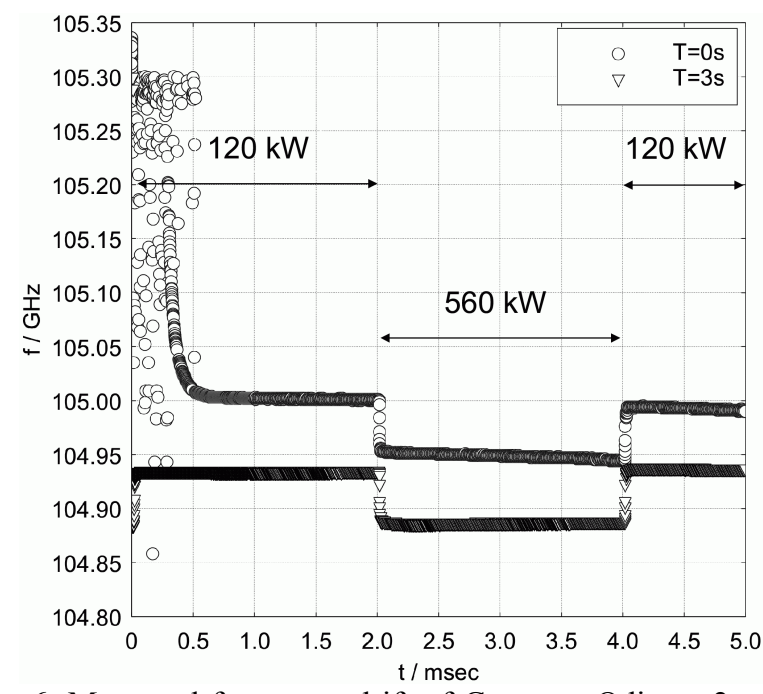

Fig. 6. Measured frequency drift of Gyrotron Odissey-2 at the beginning and after $3 \mathrm{~s}$ during a pulse with partial modulation of only the cathode voltage at $105 \mathrm{GHz}$.

\section{MATCHING OPTICS UNIT}

In step-tunable gyrotrons the output beam leaves the gyrotron window for different frequencies at slightly different azimuthal angles and positions due to the varying caustic radii of the different modes. Therefore the MOU (Fig.7) contains different sets of phase correcting mirrors (M1, M2) to match the gyrotron output beam at different frequencies to the transmission line input. The phase correcting mirrors are mounted on turntables and automatically switched into the beam path when the frequency is changed. Space requirements limit the number of required phase correcting mirror sets and therefore we chose four frequencies as our main operating modes for the step-tunable gyrotrons. The second mirror M2 contains a coupling-hole array for pulse monitoring and power measurement. Only one set of polarizers (P1, P2) with groove depths of $\lambda / 4$ and $\lambda / 8$ scaled to the center frequency of 122.5 $\mathrm{GHz}$ proved to be sufficiently broadband to provide the required range of ellipticity and orientation of the polarization ellipse for all necessary injection angles over the whole frequency band of the system $(105-140 \mathrm{GHz})$ [11]. The MOU contains also two switching mirrors that can direct the beam to a $1 \mathrm{~s}$ calorimetric load [12] which is part of each MOU, or to a central long-pulse load. Using the $1 \mathrm{~s}$ loads, all four gyrotrons can be started up simultaneously every day. 


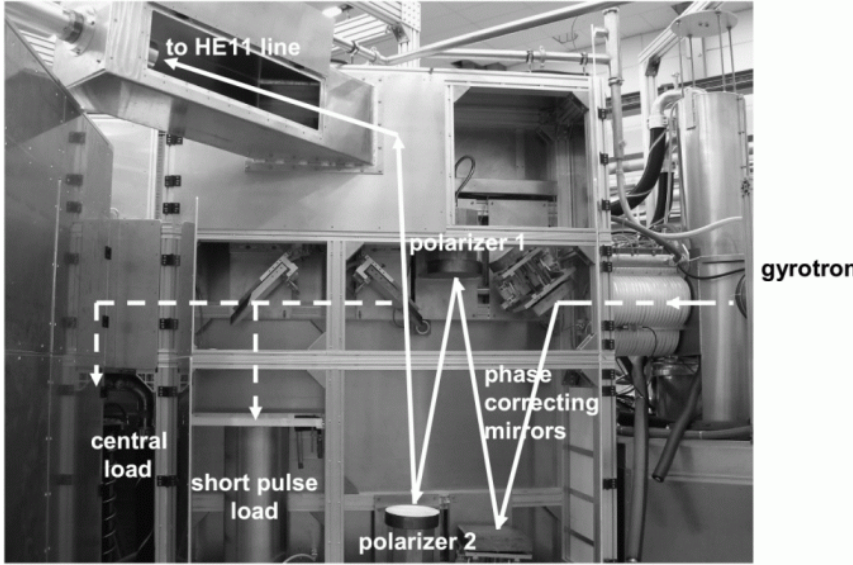

Fig. 7. Matching optics unit with phase correcting mirrors and polarizers.

\section{TRANSMISSION LINE}

The transmission to the torus is in normal air, through corrugated aluminum $\mathrm{HE}_{11}$ waveguides with $\mathrm{I} . \mathrm{D} .=87 \mathrm{~mm}$ over a total length of about $70 \mathrm{~m}$ (Fig.8). Since most part of the waveguide path is straight, the number of miter bends could be limited to eight between MOU and torus input. Two transmission lines have been installed so far. All waveguides use a common support and are optically aligned. Another calorimetric load $(0.1 \mathrm{~s})$ is installed at the end of the transmission line at the torus. This load is used to test the transmission line prior to plasma shots as well as for calorimetric measurements of the transmission efficiency. Table 2 gives the measured transmission losses for the twofrequency gyrotrons Odissey-1 and Odissey-2, which are in reasonable agreement with the theoretical predictions. The estimated error bar in the calorimetric measurements is at maximum $\pm 10 \%$. The overall losses are also sensitive to the alignment of the gyrotron output beam to the transmission line which directly affects the mode purity in the waveguide. This might be responsible for the difference in the two measurements.

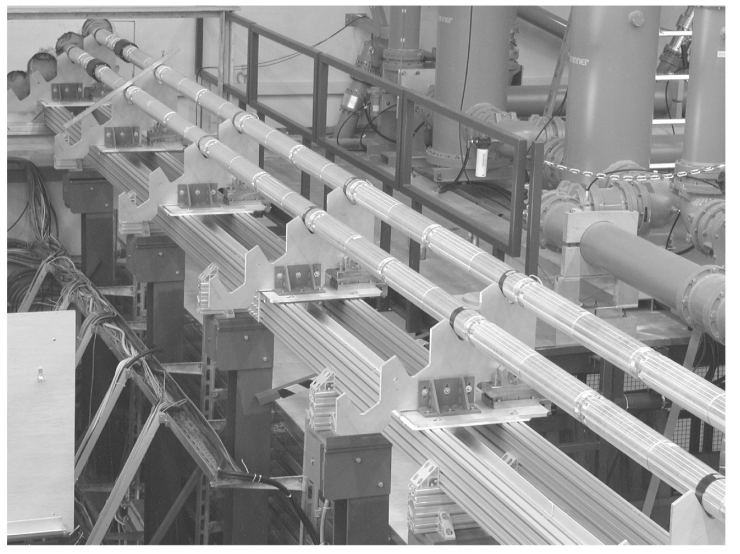

Fig. 8. Part of the $\mathrm{HE}_{11}$ transmission line.

\begin{tabular}{|l|c|c|}
\hline \multicolumn{3}{|c|}{ Estimated losses } \\
\hline Frequency & $\mathbf{1 0 5} \mathbf{~ G H z}$ & $\mathbf{1 4 0 ~ G H z}$ \\
\hline Ohmic losses (70m HE11 waveguide) & $0.12 \%$ & $0.05 \%$ \\
\hline Ohmic losses (8 miter bends) & $0.76 \%$ & $1.03 \%$ \\
\hline Diffraction losses (8 miter bends) & $5.28 \%$ & $3.43 \%$ \\
\hline Atmospheric absorption (70m) & $1.2 \%$ & $3.17 \%$ \\
\hline Total losses & $7.36 \%$ \\
\hline \multicolumn{2}{|c|}{ Measured total losses } \\
\hline Transmission line 1 with Odissey-1 & $(12.0 \pm 2.4) \%$ & $(10.0 \pm 2.0) \%$ \\
\hline Transmission line 2 with Odissey-2 & $(5.0 \pm 1.0) \%$ & $(8.0 \pm 1.6) \%$ \\
\hline
\end{tabular}

Table 2 Estimated and measured transmission losses.

\section{BROADBAND VACUUM WINDOWS}

Except for the two-frequency gyrotron, where a single-disc diamond window is transparent at both frequencies, the vacuum windows required for the step-tunable gyrotron and at the torus must be broadband. The gyrotron with its linearly polarized output beam allows the application of a Brewster window. The gyrotron Odissey-1 is currently being equipped with such a window. A gyrotron with Brewster window requires additional mirrors providing the passing of the beam through the window at the correct angle (Fig.9a). To avoid constraints with respect to polarization which is set by the two polarizers in the MOU, a tunable double-disc window with a remote controlled adjustment of the distance between the discs will be used at the torus (Fig.9b) [13]. Two diamond discs with a thickness of $1.8 \mathrm{~mm}$ will be utilized for this window, where the discs themselves are resonant at 105 and $140 \mathrm{GHz}(3 \lambda / 2$ and $4 \lambda / 2$ respectively). For intermediate frequencies the double-disc window can be tuned to a reflection minimum by changing the distance between the two discs. A critical value is the width of the Fabry-Perot resonances at intermediate frequencies between the single-disc resonances (Fig.10). Only a maximum distance of $10 \mathrm{~mm}$ between the discs can be allowed for a possible frequency drift of $140 \mathrm{MHz}$ during the gyrotron pulse to keep the reflection below the critical value of $1 \%$. Figure 11 shows the double disc window together with a comparison of the calculated reflection with the cold test measurements. The volume between the two discs will be evacuated in order to increase the power handling capability.

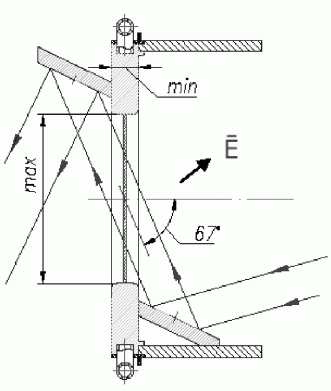

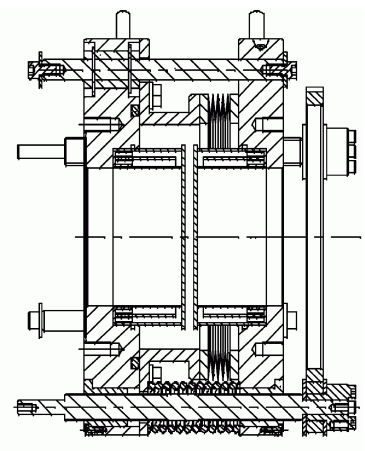

b
Fig. 9. Schematic of both broadband windows: (a) Brewster and (b) double-disc. 


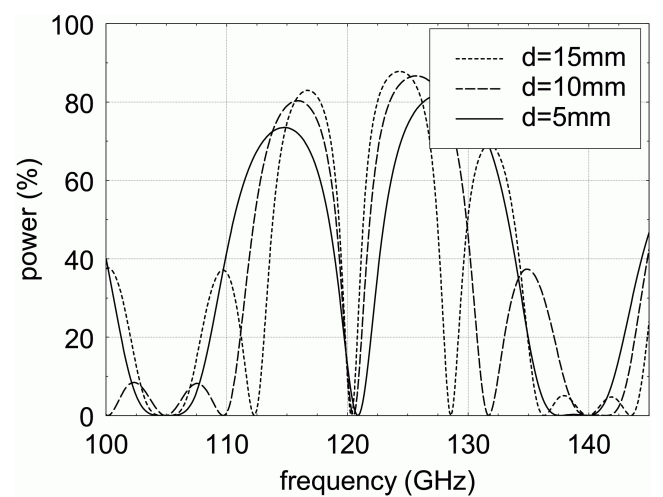

Fig. 10. Calculated reflection of a double disc window for different distances d between the discs.
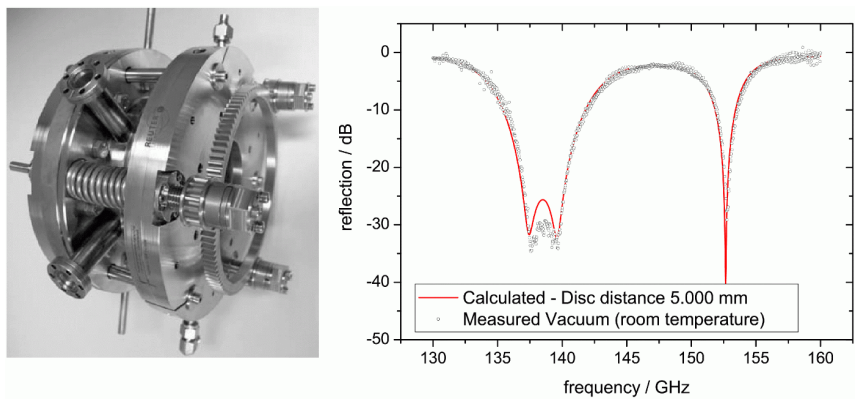

Fig. 11. Double disc torus window with cold test results.

\section{FAST STEERABLE LAUNCHER}

A steerable launcher will enable the steering of the beam over the whole plasma cross-section. In order to cope with thermal load, disruption forces and mechanical dynamics of the fast poloidal steering, the mirror is made out of high heat conductivity fine grain graphite. In order to reduce its ohmic losses it has a copper coating on the reflecting surface. High power tests at IPP Greifswald using a $750 \mathrm{~kW}$ beam at 140 $\mathrm{GHz}$ with repetitive pulses of $20 \mathrm{~s}$ proved the thermal stability of the metallic layer. The measurements are in good agreement with a numerical analysis predicting maximum surface temperatures of $350{ }^{\circ} \mathrm{C}$ during the pulse and a rise in bulk temperature of $40{ }^{\circ} \mathrm{C}$ after the pulse. Two different types of drives are used for the launcher. A slow drive rotates the launcher around its axis on a shot to shot basis, mainly to set the toroidal launching angle. A fast spindle drive controls the poloidal launching angle during a discharge (Fig.12). Figure 13 shows the mounted launchers in the ASDEX Upgrade port. In Figure 14 the result of a dynamical test of the launcher during a typical ASDEX Upgrade plasma discharge is given. The launcher movement (solid line) contains both acceleration and deceleration of the mirror as well as a phase with a constant velocity. There is also a delay in the response to the start and stop signal of the remote control (dashed line). The design value of $10^{\circ} / 100 \mathrm{~ms}$ for the fast poloidal steering was achieved during the tests. Currently two more launchers are being built into the port. This capability will allow feedback control of the deposition on the time scale of NTM growth, providing the possibility to validate this scheme for ITER in
ASDEX Upgrade.

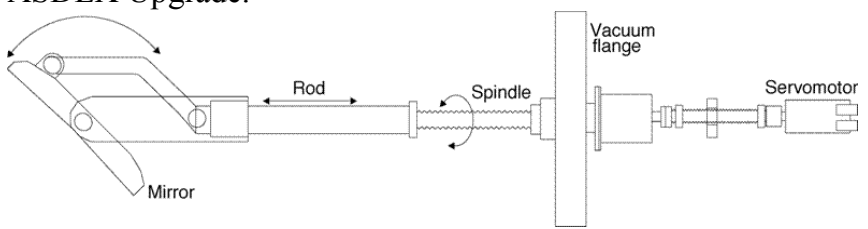

Fig. 12. Push-rod drive for the fast steerable launcher.

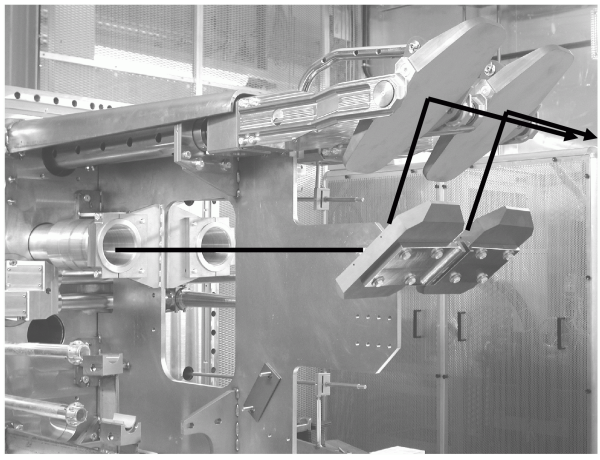

Fig. 13. Mounted launcher in the ASDEX Upgrade port.

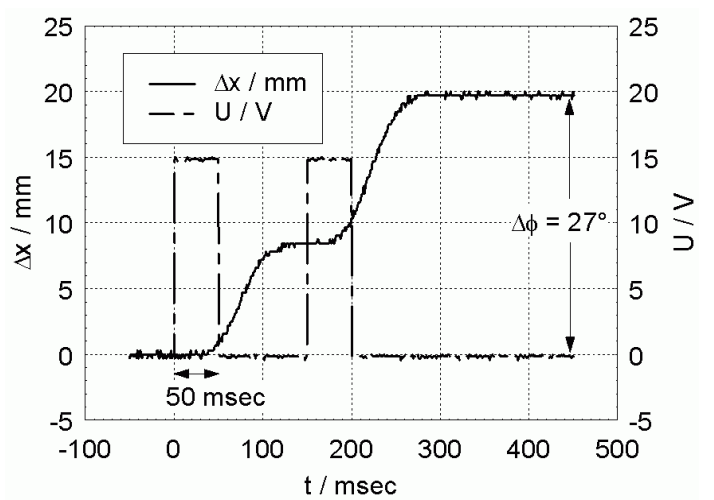

Fig. 14. Dynamical test of the fast steerable launcher during an ASDEX Upgrade shot. Dashed line: control voltage, solid line: adjustable stroke of the fast spindle drive.

\section{CONCLUSIONS}

The new multi-frequency ECRH system for ASDEX Upgrade poses new challenges not only to gyrotron development, but also to the matching optics and transmission elements, which have to be broadband. Two transmission lines are completed so far. The two-frequency gyrotrons Odissey-1 and Odissey- 2 have successfully been tested together with the first transmission line. Gyrotron Odissey-1 will be equipped with a broadband Brewster CVD-diamond output window. Together with the installation of the first broadband CVDdiamond double disc window this will allow for the operation at additional intermediate frequencies. Further extension to four gyrotrons and transmission lines is underway. A fast steerable launcher, which will be used for feedback controlled deposition, was tested under experimental conditions during plasma discharges in ASDEX Upgrade. First plasma test shots were performed with maximum power at $140 \mathrm{GHz}$ and pulse lengths of several seconds [14]. With this system, the first large 
scale multi-frequency ECRH system on a fusion device will be realized, leading to unprecedented flexibility in ECRH operation on ASDEX Upgrade.

\section{REFERENCES}

[1] H. Zohm and M. Thumm, "On the use of step-tunable gyrotrons in ITER”, J. of Phys: Conf. Series, 25, 274-282, May 2005.

[2] K.E. Kreischer and R.J. Temkin, "Single-mode operation of a highpower, step-tunable gyrotron", Physics Review Letters, 59, 547-550, August 1987.

[3] M. Thumm et al., "Frequency step-tunable (114-170 GHz) megawatt gyrotrons for plasma physics applications", Fusion Engineering and Design 53, 407-421, June 2001.

[4] F. Leuterer et al., "Plans for a new ECRH System at ASDEX Upgrade", Fusion Engineering and Design, 66-68, 537-542, September 2003.

[5] M. Maraschek et al., "Enhancement of the Stabilisation Efficiency of a Neoclassical Magnetic Island by Modulated Electron Cyclotron Current Drive in the ASDEX Upgrade Tokamak", Physics Review Letters, 98, 25005-1-4, January 2007.

[6] V. Zapevalov et al., "Development of a Prototype of a 1-MW 105-156$\mathrm{GHz}$ Multifrequency Gyrotron", Radiophysics and Quantum Electronics 47, 396-404, May 2004.

[7] P. Woskov et al., "Frequency Measurements of the Gyrotrons used for CTS Diagnostics at TEXTOR and ASDEX Upgrade", 16 ${ }^{\text {th }}$ APS High Temperature Plasma Diagnostics Conference, Williamsburg, VA, May 7 11, November 2006.

[8] G.I. Zaginaylov et al., "Influence of background plasma on electromagnetic properties of "cold" gyrotron cavity", IEEE Trans. Plasma Science, 34 (3), 512-517, June 2006.

[9] G. Nusinovich et al., Slow processes in startup scenarios of long-pulse gyrotrons", Physics of Plasmas, 13, 083016, August 2006.

[10] W. Kasparek et al., "Fast Switching and Power Combination of Highpower Electron Cyclotron Wave Beams: Principles, Numerical Results, and Experiments", Fusion Science and Technology, 52, No.2, 281-290, August 2007.

[11] Wagner D and Leuterer F, " Broadband Polarizers for High-Power MultiFrequency ECRH Systems”, Int. J. on Infrared and Millimeter Waves, 26 163-172, February 2005.

[12] F. Gandini et al., "Short-pulse Calorimetric Load for High Power Millimeter-wave Beams, Int. J. on Infrared and Millimeter Waves, 28, 121-128, February 2007.

[13] X. Yang et al., Analysis of transmission characteristic for single and double disk windows, Int. J. on Infrared and Millimeter Waves, 24, 619628, May 2003.

[14] J. Stober et al.., First experiments with the extended ECRH system on ASDEX Upgrade, $34^{\text {th }}$ EPS conference on Plasma Physics, Warsaw, Poland, July 2-7, to be published. 